\title{
MicroRNA-185 reduces the expression of hepatitis B virus surface antigen by targeting PRKCH in HepG2 2.2.15 cells
}

\author{
RONGHUA ZHANG ${ }^{1 \#}$, ZHANGXINYI LIU ${ }^{2 \#}$, XIAOLI HOU ${ }^{1}$, MEIMEI WANG ${ }^{1}$, YANAN XIONG ${ }^{1}$, HONGJIAN LU', \\ YITONG WANG ${ }^{1}$, JINGHUI SU ${ }^{1}$, YUTAN LIU ${ }^{1}$, GUANGLING ZHANG ${ }^{3 *}$, YANKUN LIU $4^{4^{*}}$
}

\begin{abstract}
${ }^{1}$ Hebei Key Laboratory for Chronic Diseases, Tangshan Key Laboratory for Preclinical and Basic Research on Chronic Diseases, School of Basic Medical Sciences, North China University of Science and Technology, Hebei, P. R.China; ${ }^{2}$ XiangYa School of Medicine, Central South University, Hunan, P. R.China; ${ }^{3}$ School of Clinical Medicine, North China University of Science and Technology, 21 Bohai Road, Tangshan, Hebei, P. R.China; ${ }^{4}$ Central laboratory of Tangshan People's Hospital, Hebei, P. R. China
\end{abstract}

\begin{abstract}
Summary. - MicroRNAs (miRNAs) are single-stranded noncoding RNAs with 18 to 25 nucleotides and play critical roles in a wide spectrum of biological processes. We repored that miR-185 inhibited hepatitis B surface antigen (HBsAg) expression and hepatitis B virus (HBV) replication without affecting the proliferation of HepG2 2.2.15 cells, compared with the controls. We identified that protein kinase C eta (PRKCH) is a direct target gene of miR-185 that affects HBV replication and protein expression and that the miR-185 may suppress HBV replication. Our results provide more information for gene therapy in HBV infection.
\end{abstract}

Keywords: miR-185; HBV; HBV surface antigen; viral replication; PRKCH

\section{Introduction}

MicroRNAs (miRNAs) are single-stranded noncoding RNAs with 18 to 25 nucleotides; endogenous miRNAs are recognized as key regulators of gene expression that act through miRNA-guided RNA silencing pathway (Ueda et al.,2010; Titov et al., 2018; Smirnova et al.,2015; Huang et al., 2018). miRNAs are generated upon processing of miRNA precursors by RNase III Dicer and are expressed in almost all studied eukaryotes (Bertoli et al., 2015). One strand from the resulting short double-stranded RNA guides the RNA-induced silencing complex to its target messenger RNA (mRNA) (Dong et al., 2017). miRNA-armed RISC can

"Corresponding authors. E-mail: zhanggl@ncst.edu.cn, phone: +8615100526789 (Guangling Zhang); yankunliu2014@yahoo. com, phone: + 8613315599558 (Yankun Liu). "Equal contribution: Ronghua Zhang \& Zhangxinyi Liu.

Abbreviations: ASO(s) = antisense oligonucleotide(s); MTS $=$ Cell Titer $96^{\circledR}$ AQueous Non-Radioactive Cell Proliferation Assay; $\mathrm{HBV}$ = hepatitis $\mathrm{B}$ virus; $\mathrm{HBsAg}$ = hepatitis B surface antigen; $\mathrm{HBeAg}=$ hepatitis $\mathrm{B}$ e-antigen; $\operatorname{miRNA}(\mathrm{s})=$ micro RNA(s); PMS = phenazine methosulfate; $\mathrm{PKC}=$ protein kinase $\mathrm{C} ; \mathrm{PRKCH}=$ protein kinase $\mathrm{C}$ eta; $\mathrm{RT}-\mathrm{PCR}=$ real-time PCR degrade mRNA in perfect sequence complementarity or inhibit mRNA translation in imperfect sequence complementarity (Ghoshal et al., 2015). Mechanisms underlying the gene regulation by miRNAs include regulation of cellular differentiation, proliferation, and apoptosis ( $\mathrm{Li}$ et al., 2013; Agarwal et al., 2015). MiR-185, which was first described as a regulator of cancer progression, is localized at chromosome 22q11.21. Results of previous studies revealed the connection between miR-185 and liver cancer (Zhi et al., 2013). Li and colleagues found that miR-185 was significantly downregulated in the plasma of patients with hepatitis B virus (HBV)-related liver fibrosis and in liver tissues of mice with $\mathrm{CCl}_{4}$-induced fibrosis (Zhou et al., 2018). In another research, they propose that miRNA pathways control the replication of both RNA and DNA viruses (Koch et al., 2014). Comprehensive research on host-pathogen interactions at microRNA level is still in its early stages. However, exploring possible interactions in certain major pathogenic viruses in humans has attracted researchers' interest.

Current experiments and computational approaches have suggested the existence of miRNAs in many viruses, such as EBV and adenoviruses (O'Connell et al., 2016). 
Recently, certain host-encoded miRNAs modulated viral replication conversely, for example, liver-specific miR-122 positively regulated the replication of hepatitis $C$ virus (Takaki et al., 2015). Impaired translation of target sequences from primate foamy virus 1 (PFV) by miR-32 has been reported (Lecellier et al., 2005). In a recent study, at least five miRNAs that were expressed in human $\mathrm{T}$ cells conserved predicted target sites within nef and virus protein $\mathrm{R}$ transcripts of human immunodeficiency virus (HIV), thereby indicating a host-pathogen interaction (Khairkhah et al., 2018). Emerging evidence on miRNAmediated crosstalk in viral infections offers opportunities to understand the intricacies of host-pathogen interactions, possible explanations to viral tropism, latency, and oncogenesis, as well as development of novel biomarkers and therapeutics.

Chronic HBV infection is a major cause of liver fibrosis, which eventually leads to cirrhosis and hepatocellular carcinoma. HBV is a hepatotropic, partially double-stranded DNA virus, whose genome presents four overlapping open reading frames that code for HBV surface antigen, core protein, X protein ( $\mathrm{HBx}$ ), and reverse DNA polymerase (Arzumanyan et al., 2013). The virus integrates its genetic information into the genome of the host cell. Our previous research showed that miR-370 suppresses HBV gene expression and replication by repressing NFIA expression, which stimulates HBV replication through direct regulation on HBV Enhancer I activities (Fan et al., 2016). miR-199a-3p and miR-210 inhibited HBsAg expression and HBV replication without inhibiting cell proliferation (Zhang et al., 2010). However, mechanisms involved in the interaction of persistent HBV infection and host cells is not well elucidated. Complex regulatory networks operated by miRNAs have not been assessed comprehensively in HBV replication.

We reported that human miRNAs can inhibit HBV replication by targeting cellular proteins such as protein kinase $\mathrm{C}$ eta (PRKCH), which is important during the replication cycle of the virus. Evidence for an intricate physiological interplay between the cell's miRNAs and HBV was provided. Our study implied that human miRNAs have the potential to affect the expression of HBV genes and may be used to develop therapeutic approaches to inhibit HBV.

\section{Materials and Methods}

Reagents. Dulbecco's modified Eagle's medium (DMEM), MEM- $\alpha$, and fetal bovine serum (FBS) were purchased from GIBCO (USA). miRNA antisense oligonucleotides (ASOs) used in the study are exact antisense copy of human mature miRNA sequence that can be found in miRNA register (Jae-Sang et al., 2015) and synthesized by IDT (USA). Lipofectamine 2000 transfection reagent and opti-MEM medium were purchased from Invitrogen (USA). SYBR green PCR master mix kit was purchased from Applied Biosystems (USA). TaqMan miRNA assay kits, real time primer, PCR primers, and TaqMan probes for miR-185 were purchased from ABI (USA). SYBR ${ }^{\circledR}$ Premix Ex Taq ${ }^{\mathrm{TM}}$ was provided by TaKaRa Bio Inc. (Japan). MTS/PMS at a final concentration of $333 \mu \mathrm{g} / \mathrm{ml}$ MTS and $25 \mu \mathrm{M}$ PMS and M-MLV kit were purchased from Promega (China). ELISA kit was purchased from InTec Products (China). mirVana ${ }^{\mathrm{TM}}$ miRNA isolation kit was purchased from Ambion (USA). MICROMAX ${ }^{\mathrm{TM}}$ TSA $^{\mathrm{TM}}$ labeling and detection kit was purchased from Perkin Elmer (USA). Ratio immunoprecipitation assay lysis buffer (RIPA lysis buffer, $50 \mathrm{mmol} / \mathrm{l}$ Tris- $\mathrm{HCl} \mathrm{pH} \mathrm{7.2,} 150 \mathrm{mmol} / \mathrm{l} \mathrm{NaCl}$, $1 \%$ Triton X-100, 0.1\% SDS), antibodies to PRKCH, $\beta$-tubulin, and horseradish peroxidase (HRP)-conjugated anti-rabbit IgG secondary antibodies were purchased from Sigma-Aldrich (USA).

Cell culture and treatment. HepG2 2.2.15 cell line, which was derived from a clone of HepG2 cells transfected with a dimer from complete HBV genome (Wei et al., 2013), was used. In addition, human hepatoblastoma cell line HepG2 and HEK293 cell line derived from human embryonic kidney were used. HepG2 2.2.15 and HepG2 cells were propagated and maintained in DMEM supplemented with 10\% FBS, 20 mmol/l HEPES, 2 $\mathrm{mmol} / \mathrm{l}$ glutamine, and antibiotics in a humidified atmosphere at $37^{\circ} \mathrm{C}$ with $5 \% \mathrm{CO}_{2}$. HEK293 cells were grown in MEM- $\alpha$ supplemented with $10 \% \mathrm{FBS}, 20 \mathrm{mmol} / 1 \mathrm{HEPES}, 2 \mathrm{mmol} / \mathrm{l}$ glutamine, and antibiotics in humidified atmosphere at $37^{\circ} \mathrm{C}$ with $5 \% \mathrm{CO}_{2}$.

Transfection. HepG2 2.2.15 cells were trypsinized and plated with $1 \times 10^{4}$ cells per well in 96 -well plates the day before transfection to ensure a suitable cell confluence during transfection. For transfection, Lipofectamine 2000 was used in accordance with the manufacturer's protocol to transfect HepG2 2.2.15 cells in 96-well plates at $60 \%-70 \%$ confluence with inhibitors or precursors of human miRNAs, $200 \mathrm{nM}$ oligonucleotides, and $5 \mathrm{ng} / \mu \mathrm{l}$ small interfering RNAs (siRNAs) plasmids in antibiotic free Opti-MEM medium. PRKCH inhibition was performed using siRNA that targets exons of PRKCH (siRNA 3544; sense: GGUCUUCACCUCAGACACUtt, antisense: AGUGUCUGAG GUGAAGACCtg; Ambion). Each experiment in this study was performed at least thrice.

Quantitative real-time $P C R$. After $72 \mathrm{~h}$ post transfection with miRNA ASOs or precursors, supernatants of HepG2 2.2.15 were harvested and used to quantify HBV DNA by RT quantitative PCR through SYBR green PCR master mix kit and ABI-Prism 7300 system. We used glyceraldehyde 3-phosphate dehydrogenase (GAPDH) as internal control. For quantification, relative HBV DNA copy was calculated as the copy ratios of HBV DNA normalized to control group.

Detection of mature miR-185 by TaqMan RT-PCR. TaqMan miRNA assays used stem loop method (Wang et al., 2018a,b) to detect the expression level of mature miR-185. For RT reactions, $10 \mathrm{ng}$ of total RNA was used in each $15 \mu$ l reaction and mixed with 
$3 \mu \mathrm{RT}$ primer. $\mathrm{RT}$ reaction was conducted under the following conditions: $16^{\circ} \mathrm{C}$ for $30 \mathrm{~min}, 42^{\circ} \mathrm{C}$ for $30 \mathrm{~min}, 85^{\circ} \mathrm{C}$ for $5 \mathrm{~min}$, and held at $4^{\circ} \mathrm{C}$. After RT reaction, 150x diluted cDNA products and $1.33 \mu \mathrm{l}$ of the diluted cDNA was used for PCR reaction with $2 \mu \mathrm{l}$ TaqMan primers. PCR reaction was conducted at $94^{\circ}$ for $5 \mathrm{~min}$, followed by 40 cycles of $94^{\circ} \mathrm{C}$ for $30 \mathrm{~s}, 50^{\circ} \mathrm{C}$ for $30 \mathrm{~s}$, and $72^{\circ} \mathrm{C}$ for $40 \mathrm{~s}$ in ABI 7500 RT-PCR system. Real-time PCR results were analyzed and evaluated as relative miRNA expression of CT (threshold cycle) value and fold change was determined (Wang et al., 2018b). U6 was used for normalization.

MTS assay. HepG2 2.2.15 cells were seeded in 96-well plates at a density of 10,000 cells per well and then transfected with miRNA ASO or precursor after $24 \mathrm{~h}$. After $72 \mathrm{~h}$ post transfection, the cells containing $100 \mu \mathrm{l}$ of culture media were incubated with $20 \mu \mathrm{l}$ of the combined MTS/PMS solution at $37^{\circ} \mathrm{C}$ for $4 \mathrm{~h}$ in a humidified, $5 \% \mathrm{CO}_{2}$ atmosphere. Absorbance at $490 \mathrm{~nm}$ was recorded using Quant Universal Microplate Spectrophotometer (Bio-tek Instruments).

ELISA for HBsAg and HBeAg detection. After $72 \mathrm{~h}$ post transfection with miRNA ASOs or precursors, supernatants of HepG2 2.2.15 were harvested and diluted in phosphate-buffered saline in a ratio of 1:9. Dilutions of the supernatants were then examined in ELISA to determine the concentrations of HBsAg and $\mathrm{HBeAg}$ using ELISA kits. Absorbance was determined in a microtiter plate reader (Quant, Biotek, Germany) with dualwavelength measurement $(450 / 630 \mathrm{~nm})$.

RNA extraction and CDNA microarray analysis. RNA extraction of cells was performed using mirVana ${ }^{\mathrm{TM}}$ miRNA isolation kit. Integrity of large RNAs was confirmed on $1 \%$ denatured agarose gel electrophoresis. Differently expressed genes between HepG2 2.2.15 cells transfected with miR-185 ASOs and with control oligonucleotides were detected on cDNA microarray using MICROMAX ${ }^{\mathrm{TM}} \mathrm{TSA}^{\mathrm{TM}}$ labeling and detection kit according to the manufacturer's instructions. Labeled cDNA was then hybridized overnight in the microarray, which contained 7267 human gene probes with each probe in duplex. Microarray was sequentially incubated with anti-FL-HRP and streptavidin-HRP, which catalyzed the deposition of Cy3 and Cy5, respectively. The slide was scanned using Packard Biochip Technologies' ScanArray ${ }^{\circledR}$ Express microarray acquisition system (Perkin Elmer).

Bioinformatics methods. miRNA targets predicted by computer-aided algorithms were obtained from PicTar (http://pictar. bio.nyu.edu/cgi-bin/PicTar_vertebrate.cgi), TargetScan Release 4.0 (http://www.targetscan.org), miRanda (http://www.microrna.org), and ViTa (http://vita.mbc.nctu.edu.tw).

Vector construction. To construct the enhanced green fluorescence protein (EGFP) reporter vector, we amplified the EGFP coding region from $\mathrm{pEGFP-N2}$ (Clontech) by PCR using primers EGFP sense and EGFP antisense with restriction sites of HindIII and BamHI (Table 1). The amplified fragment was cloned to pcDNA3.1(+) (Invitrogen) at HindIII and BamHI sites. PRKCH 3'-UTR fragment containing the predicted miR-185 binding site was amplified by PCR using primers PRKCH sense and PRKCH antisense with EcoRI and XhoI site (Table 1). The amplified fragment was cloned to pcDNA3.1(+)/EGFP at EcoRI and XhoI sites downstream of EGFP coding region. The constructed vector was named pcDNA3/EGFP-PRKCH UTR. Similarly, PRKCH 3'UTR mutant fragments, which contained mutational miR-185 binding sites, were cloned to the pcDNA3.1(+)/EGFP at the same sites. The constructed vector was named pcDNA3/EGFP-PRKCH UTR mut. To construct a plasmid-expressing miR-185, we amplified a 582-bp DNA fragment carrying pri-miR-185 from HEK293 genome using PCR primers, pri-miR-185 sense, 5'-CGGGATC CAAAAGGACAAGGACT-3', where BamHI site is underlined, and pre-miR-185 antisense, 5'-GGAATTCTACACGTAGGACA GACAG-3', where the EcoRI site is underlined. The amplified fragment was cloned into pcDNA3B at BamHI and EcoRI sites. The constructed vector was named pcDNA3B/pri-miR-185. All PCR products were verified by DNA sequencing before cloning into the final destination vector.

Fluorescent reporter assay. HepG2 2.2.15 and HEK293 cells were seeded in 48-well plate the day before transfection. Cells were transfected with miR-185 ASOs or precursors and then with reporter vectors on the next day. RFP expression vector pDsRed2-N1(Clontech) was spiked and used for normalization. Cells were lysed with RIPA lysis buffer after $48 \mathrm{~h}$, and proteins

Table 1. Primers used in the study

\begin{tabular}{lll}
\hline Name & Primer 5'-3' & Restriction site \\
\hline EGFP sense & GCAGCCAAGCTTGCCACCATGTGTAGCAAGGGC & HindIII \\
EGFP antisense & CGCGGATCCTTTACTTGTACAGCTCGTCC & BamHI \\
PRKCH sense & CGGAATTCGGTATCTCTGAAACTCACAACC & EcoRI \\
PRKCH antisense & CAGTCCTCGAGGTGATGAGCACACGGCGATACAG & XhoI \\
ATP5B sense & CGGAATTCAAGTTGACCGTGTCCC & - \\
ATP5B antisense & ACCACTCGAGCAATCAAGGCTCTTGTG & - \\
NR1D1 sense & CTGTGCGGGAGGTGGTAGAGTTTGC & - \\
NR1D1 antisense & TGCCGCTCGAGGGAGGCAGGTATTTAC & - \\
$\beta$-actin sense & CGTGACATTAAGGAGAAGCTG & - \\
$\beta$-actin antisense & CTAGAAGCATTTGCGGTGGAC & - \\
\hline
\end{tabular}


were harvested. Intensities of EGFP and RFP fluorescence were detected in Fluorescence Spectrophotometer F-4500 (HITACHI).

Semiquantitative RT-PCR. Approximately $5 \mu \mathrm{g}$ of large RNA extracted from HepG2 2.2.15 cells transfected with miR185 ASOs or control oligonucleotides, miR-185 precursor, or control precursor, were transcribed to cDNA using reverse transcriptase M-MLV (Promega). cDNA was used for the amplification of PRKCH, ATP5B and NR1D1, which were candidate target genes of miR-185, endogenous control gene $\beta$-actin. PCR cycles were as follows: initial denaturation at $95^{\circ} \mathrm{C}$ for $5 \mathrm{~min}$, followed by 35 cycles of $94^{\circ} \mathrm{C}$ for $1 \mathrm{~min}, 56^{\circ} \mathrm{C}$ for $1 \mathrm{~min}, 72^{\circ} \mathrm{C}$ for $1 \mathrm{~min}$, and final extension at $72^{\circ} \mathrm{C}$ for $8 \mathrm{~min}$. PCR primers used for PRKCH, ATP5B, NR1D1 and $\beta$-actin are in the Table 1. All PCR products were resolved in $2 \%$ agarose gel. LabWorks ${ }^{\mathrm{TM}}$ Image Acquisition and Analysis Software (UVP) was used to quantify band intensities.

Western blot analysis. HepG2 2.2.15 cells were transfected with miR-185 ASOs or control oligonucleotides or pcDNA3B/ pri-miR-185 or control vector. After $48 \mathrm{~h}$, the cells were lysed with RIPA lysis buffer, and proteins were harvested. Proteins were resolved in SDS-denatured polyacrylamide gel and transferred onto a nitrocellulose membrane. Antibodies to PRKCH (Saierbio Inc.) and $\beta$-tubulin (Sigma-Aldrich) were incubated with the membrane at $4^{\circ} \mathrm{C}$ overnight. Membranes were washed and incubated with HRP-conjugated anti-rabbit IgG secondary antibodies (Sigma-Aldrich). Enhanced chemiluminescence was obtained, and the membranes were exposed to the X-ray film (Fujifilm). LabWorks ${ }^{\mathrm{TM}}$ Image Acquisition and Analysis Software (UVP) was used to quantify band intensities.

Statistical analysis. Statistical significance was determined using two-tail homoscedastic Student t test. For all data analyzed, significance threshold of $\mathrm{P}<0.05$ was assumed. In all figures, values are expressed as mean \pm standard deviation, and statistical significance is indicated by a single $(\mathrm{P}<0.05)$ or double $(\mathrm{P}<0.01)$ asterisk. Data generated in vitro are representative of at least three distinct experiments conducted in triplicate.

\section{Results}

HBsAg expression and HBV replication were increased by antisense oligomers of miR-185

Special miRNA ASO was used to block the function of endogenous miRNAs to identify which miRNAs influenced HBV replication. A total of 328 human miRNA ASO were transfected into HepG2 2.2.15 cells. ASO of LacZ was used as the control. At $72 \mathrm{~h}$ post transfection, HBsAg and $\mathrm{HBeAg}$ levels in the culture supernatant were assessed by ELISA, and cell proliferation was measured using MTS. miRNA ASO that affected the expression of HBV antigen but had no effect on cell viability were chosen for further study. In comparison with controls, transfection of 200 $\mathrm{nM}$ antisense oligomers of miR-185 elevated HBsAg expression by 1.6-fold, which was not due to the promotion of cell proliferation, as shown in Fig. 1a. Furthermore, inhibition of miR-185 had no effect on HBeAg expression (Fig. 1a). We detected HBV DNA as the amount of virus present in supernatant of transfected HepG2.2.2.15 cells (Fig. 1b) using RT-PCR. The antisense oligomers of miR-185 can increase the generation of HBV.

\section{HBsAg expression and $H B V$ replication were inhibited by overexpression of miR-185}

The involvement of miR-185 in controlling HBV replication and HBsAg expression was investigated. We constructed plasmids that express miR-185 precursors by inserting fragments containing precursor sequences of miR-185 amplified by PCR into pcDNA3B vector (pcDNA3B/pri-miR-185). Real-time PCR was used to detect miRNA levels in cells after antisense oligomer blocking and overexpression by plasmids. Ectopic expression of miR-185 plasmids was increased by 4.5-fold compared with the vector control, as indicated in Fig. 1f. However, specific antisense oligomers had minimal reduction of miR-185 level, using the same method (Fig. 1e). We further transfected pcDNA3B/pri-miR-185 plasmids into HepG2 2.2.15 cells to examine the regulation of HBV generation and antigen expression by miR-185 overexpression. ELISA and RT PCR assay showed that the ectopic expression of miR- 185 resulted in a 40\%-50\% reduction of HBsAg expression and suppression of $\mathrm{HBV}$ replication but had no effect on cell proliferation (Fig. 1c and 1d). Transfection with miR-185 ASO enhanced HBsAg mRNA expression level (Fig. 1g), compared with the control. Conversely, miR-185 overexpression reduced HBsAg mRNA expression level (Fig. 1g). These observations suggested that miR-185 inhibited HBsAg expression and HBV replication.

\section{Identification of candidate target genes of $\operatorname{miR}-185$}

To further analyze the mechanisms underlying the control of HBsAg expression and HBV replication by miR185, four well-established microRNA target prediction software, namely, miRanda (Eric et al., 2010), TargetScan (Miyaki et al., 2014), PicTar (Hausser et al., 2014), and ViTa (Nan et al., 2017) were used to predict targets for miR-185. miR-185 did not target the HBV genome directly, thereby suggesting that it might have affected HBV replication by targeting cellular protein(s). Many putative miR-185 targets were predicted by various computer-aided algorithms. However, the predicted target genes were in large quantity and most of them were not experimentally vali- 
(a)

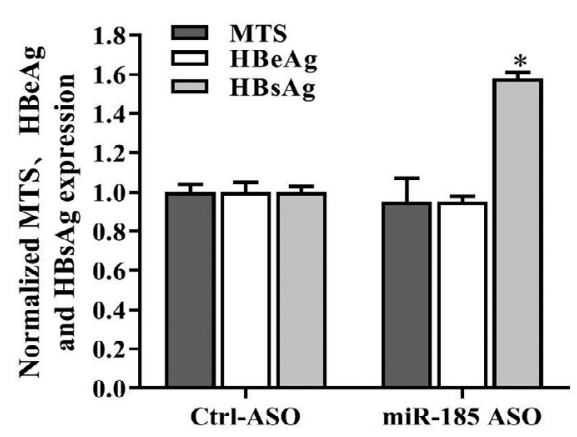

(d)

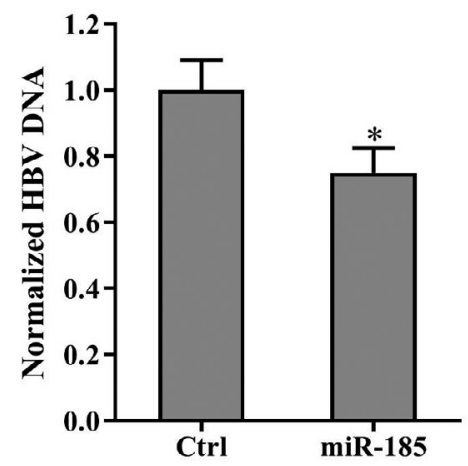

(g)

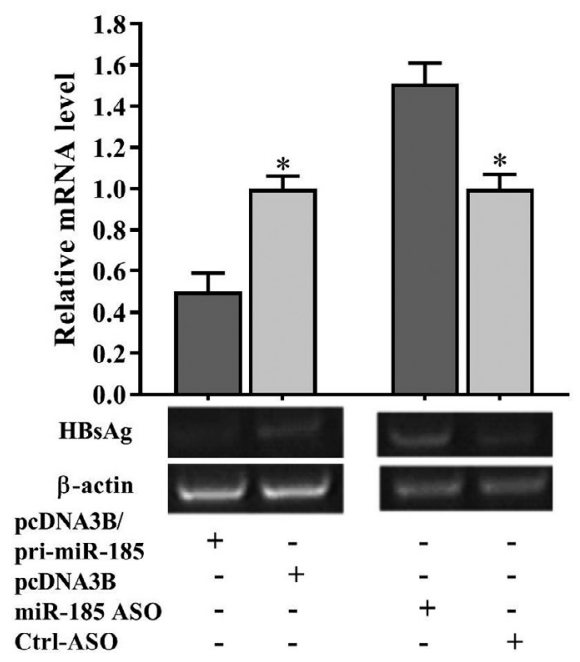

(b)

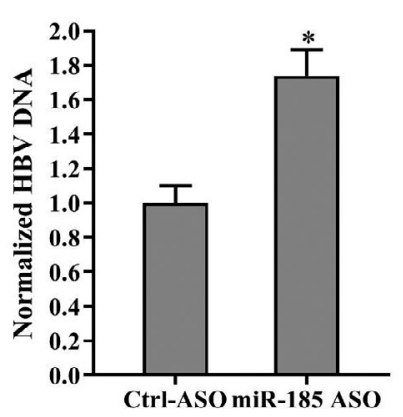

(e)

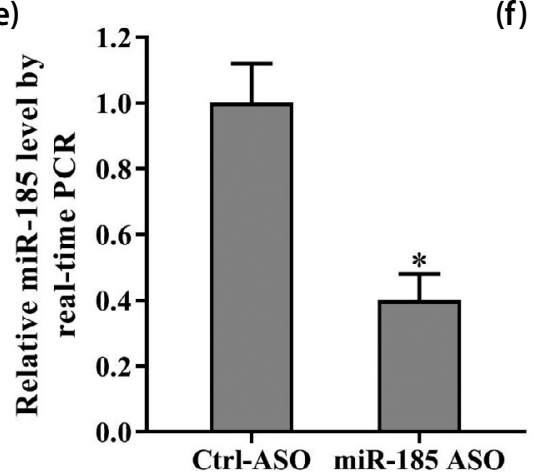

(c)

(f)
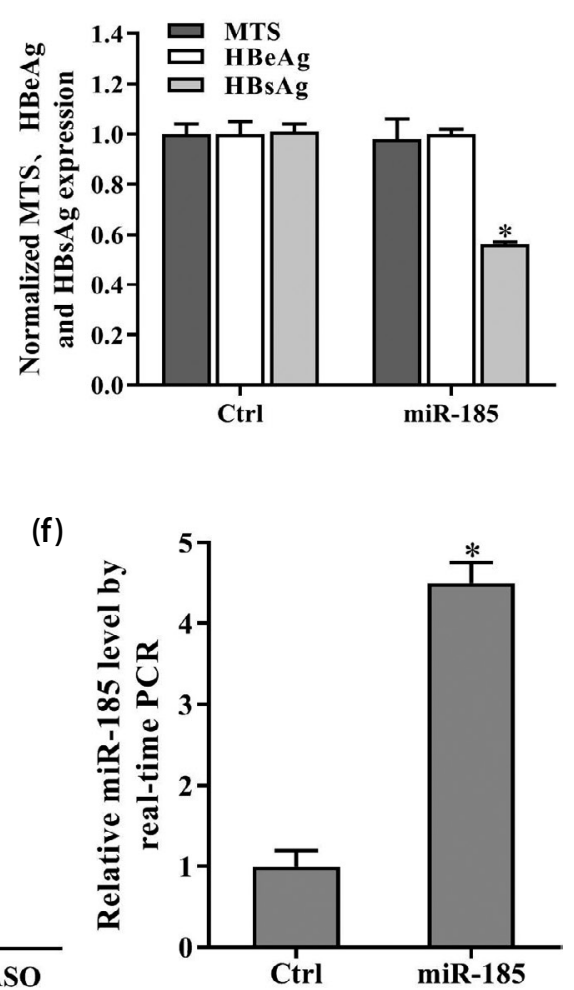

Fig. 1

miR-185 inhibited HBsAg expression and HBV replication in HepG2 2.2.15 cells

HepG2 2.2.15 cells were transfected with miRNA precursors or control vector or miRNA ASO or control ASO. HBsAg and HBeAg levels were detected by ELISA and cell proliferation was tested by MTS (a, c). HBV DNA levels were analyzed by RT-PCR in HepG2 2.2.15 cells (b, d). MiR-185 expression levels were assessed by RT-PCR (e, f) and the level of HBsAg mRNA was determined by semi-quantitative RT-PCR (g). Data were expressed as mean $\pm S D(n=3)$. ${ }^{*}<<0.05$ versus control.

results were confirmed by semi-quantitative RT-PCR. Consensus sets in figure 2 from these two methods produced the candidate miR-185 targeted gene, $\mathrm{PRKCH}$.

\section{Validation of candidate target genes of miR-185}

We amplified PRKCH 3'-UTR region, in which the predicted miR-185 binding site from HEK293 cells was 
(a)

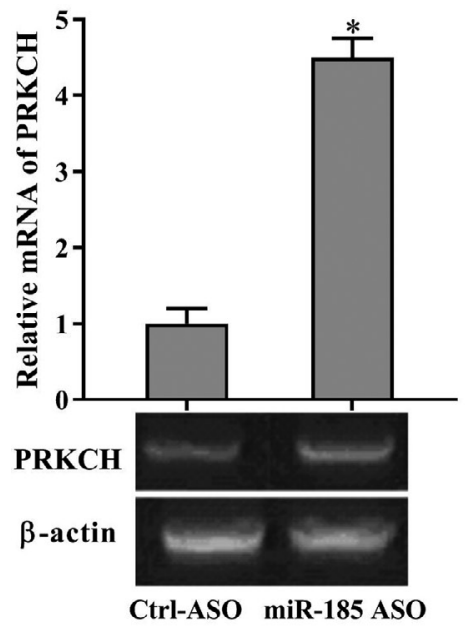

(b)

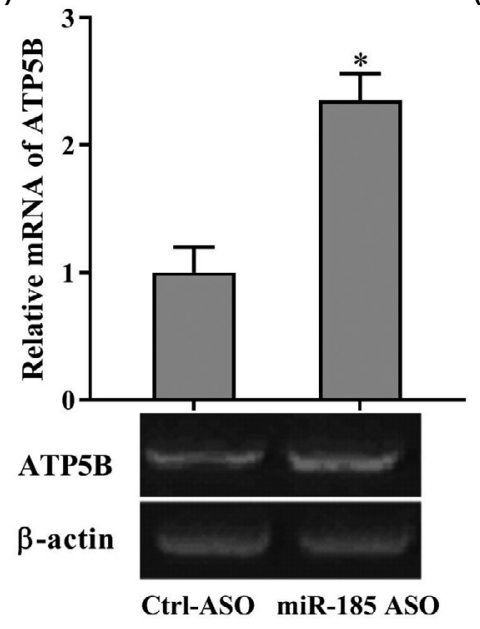

(c)

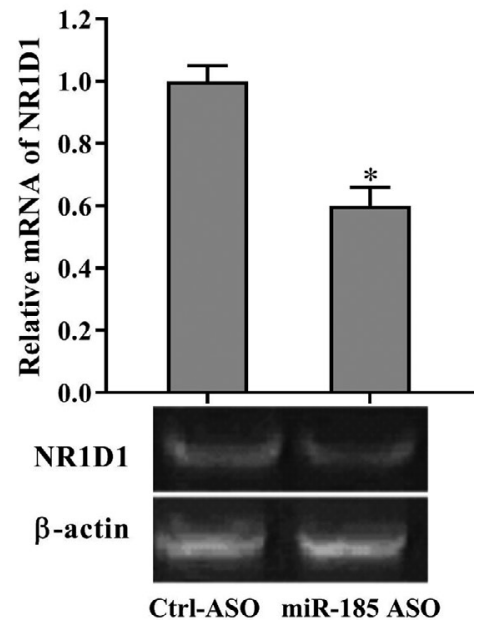

Fig. 2

Confirmation of candidate target genes of miR-185 screened in HepG2 2.2.15 cells

HepG2 2.2.15 cells were transfected with either miR-185 ASO or control ASO. At $48 \mathrm{~h}$ post transfection, mRNA levels of PRKCH (a), ATP5B (b), and NR1D1 (c) were determined by semi-quantitative RT-PCR. Data were expressed as mean $\pm \mathrm{SD}(\mathrm{n}=3)$. " $\mathrm{P}<0.05$ versus control.

located for further characterization of PRKCH as a target for miR-185. Hence, we cloned 3'-UTR into pcDNA3.1(+)/ EGFP vector. The predicted results were confirmed by EGFP reporter vectors with the intact putative miRNAs recognition sequence from PRKCH 3'-UTR or mutated seed sequences cloned downstream of EGFP gene (Fig. 3a). HepG2 2.2.15 cells were co-transfected with pcDNA3.1(+)/ EGFP-PRKCH-UTR and pcDNA3.1(+)/EGFP-PRKCH-UTR mut with or without miRNA precursors and with or without miRNA ASOs. Cells were lysed after $72 \mathrm{~h}$ and proteins were harvested. GFP and RFP activity were measured $48 \mathrm{~h}$ after transfection. Results were represented as the normalized ratio of GFP to RFP (Figs. 3b, 3c, and 3d). These results indicated that miR-185 interfered with PRKCH mRNA via direct interaction with 3'-UTR.

\section{miR-185 regulate $P R K C H$ by $m R N A$ degradation}

Total RNA and proteins were isolated from HepG2 2.2.15 cells transfected with miR-185 ASO or control ASO to determine the effect of miR-185 on PRKCH expression levels. mRNA and protein level of PRKCH were quantified using semi-quantitative RT-PCR and Western blot analysis, respectively. The mRNA and protein levels of PRKCH were elevated in HepG2 2.2.15 cells transfected with miR185 ASO compared with cells transfected with control ASO (Fig. 4a,b). Simultaneously, HepG2 2.2.15 cells were transfected with miR-185 expressing vector (pcDNA 3B/ pri-miR-185) or control vector (pcDNA3B). PRKCH mRNA and protein levels were assessed $48 \mathrm{~h}$ post transfection.
The overexpression of miR-185 in HepG2 2.2.15 cells considerably reduced the expression of PRKCH at $\mathrm{MRNA}$ and protein levels (Fig. 4a,b).

\section{Discussion}

miRNAs, which are processed from noncoding regions of the genome into 18-25 nucleotide long single-stranded RNA, regulate translation of mRNA by binding to it and implementing target cleavage or translational block depending on the extent of sequence complementarity with the target (Titov et al., 2018). miRNA-mediated regulation encompasses a wide spectrum of host biological processes ranging from growth and development to oncogenesis (Liu et al., 2018; Lau et al., 2014). Recent reports also elucidated the role of miRNAs as critical effectors in the intricate host-pathogen interaction networks (Bertoli et al., 2015).

To identify which miRNAs influence HBV replication, we utilized HepG2 2.2.15 cells, which constitutively expressed $\mathrm{HBs}$ (HBsAg), $\mathrm{HBe}$ (HBeAg), and HB core (HBcAg) antigens and supported full HBV replication (Wei et al., 2013). This in vitro system can be used to study the life cycle of HBV and the interaction of immunocompetent cells with cells carrying HBV. Results presented in this study showed that miR-185 indirectly inhibited $\mathrm{HBV}$ replication and HBsAg expression by targeting PRKCH, which is a member of the family of Protein kinase C (PKC) (Newton., 2018). PRKCH also represents the serin/threonine kinase 
(a)

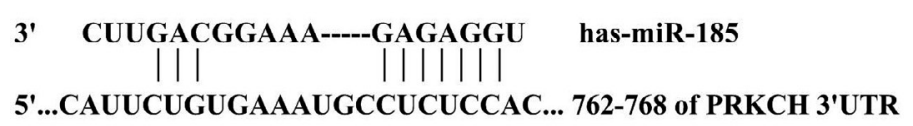

3' CUUGACGGAAA----GAGAGGU has-miR-185

5'...CAUUCUGUGAAAUGCCACACGAC... 762-768 of PRKCH 3'UTRmut

(c)

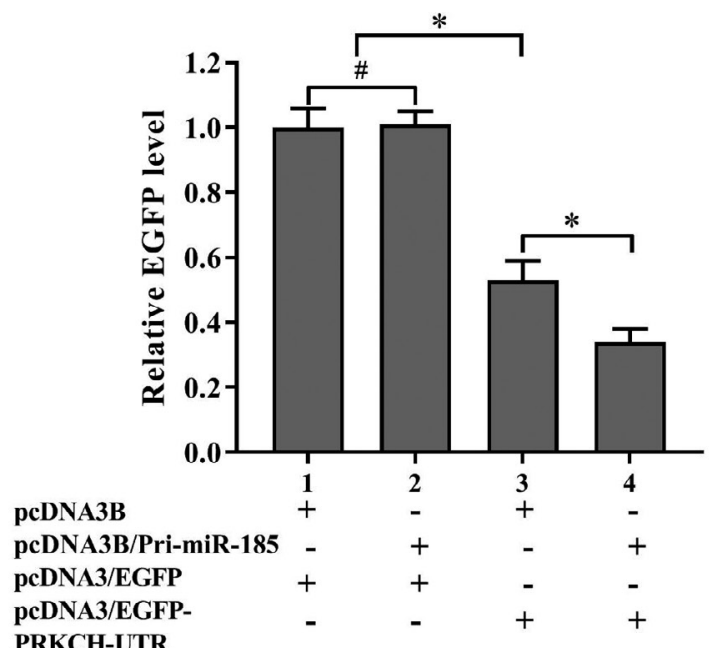

(b)

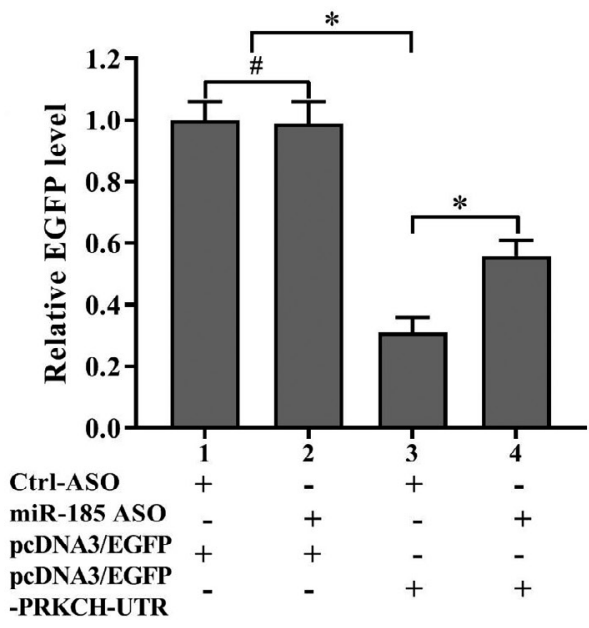

(d)

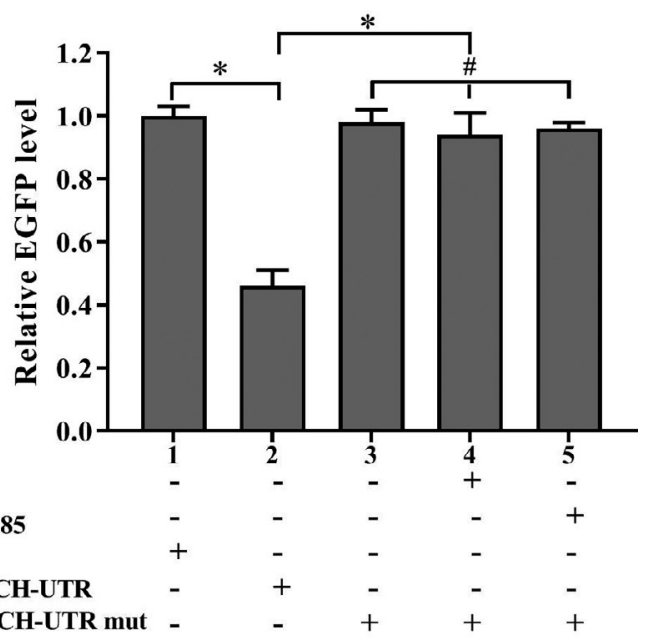

mik-185 ASO pcDNA3B/Pri-miR-185 PCDNA3/EGFP pcDNA3/EGFP-PRKCH-UTR
pcDNA3/EGFP-PRKCH-UTR mut

Fig. 3

Validation of PRKCH as a target of miR-185 in HepG2 2.2.15 cells

Complementary sequences of PRKCH mRNA3'-UTRs or a mutated form to miR-185 (a). EGFP expression of reporter vectors bearing PRKCH mRNA 3'-UTR sequences after co transfection with miR-185 ASO (b) or pcDNA3B/Pri-miR-185 (c) in HepG2 2.2.15 cells. EGFP expression of reporter vectors bearing PRKCH mRNA 3'-UTR mut sequences after co transfection with miR-185 ASO or pcDNA3B/Pri-miR-185 in HepG2 2.2 .15 cells $(\mathbf{d})$. Data were expressed as mean $\pm \mathrm{SD}(\mathrm{n}=3)$. ${ }^{*} \mathrm{P}<0.05,{ }^{*} \mathrm{P}>0.05$ versus control.

family, which regulates cell growth, differentiation, and transformation (Park et al., 2014). Previous studies had shown that miR-24-3p promoted the p53/p21 pathway by down-regulating PRKCH expression in lacrimal adenoid cystic carcinoma cells (Zhang et al., 2016). In contrast to the effects of miR-185 on HBsAg levels, HBeAg levels did not change when miR-185 was blocked or overexpressed. Zhang and colleagues had revealed that miR-199a-3p and miR-210 inhibited HBsAg expression and HBV proliferation without inhibition of cell growth (Zhang et al., 2010). miRNA, which regulates the interaction between host cells and HBV replication, plays an important role in HBV proliferation.
Identification of miRNA target genes may identify their functions. Thus, we adopted cDNA microarray analysis and used bioinformatics to predict miRNA targets. We validated by ELISA and RT-PCR that the overexpression of miR-185 can actively inhibit HBV replication and HBsAg expression. Conversely, transfection with corresponding ASO increased HBV DNA copy and HBsAg expression. We implemented a consensus target-prediction approach using multiple miRNA target prediction software, miRanda, TargetScan, PicTar, and ViTa to further analyze the mechanisms underlying miR-185's control of HBsAg expression and HBV replication. These softwares examine the base pairing of miRNA and target, thereby emphasiz- 
(a)

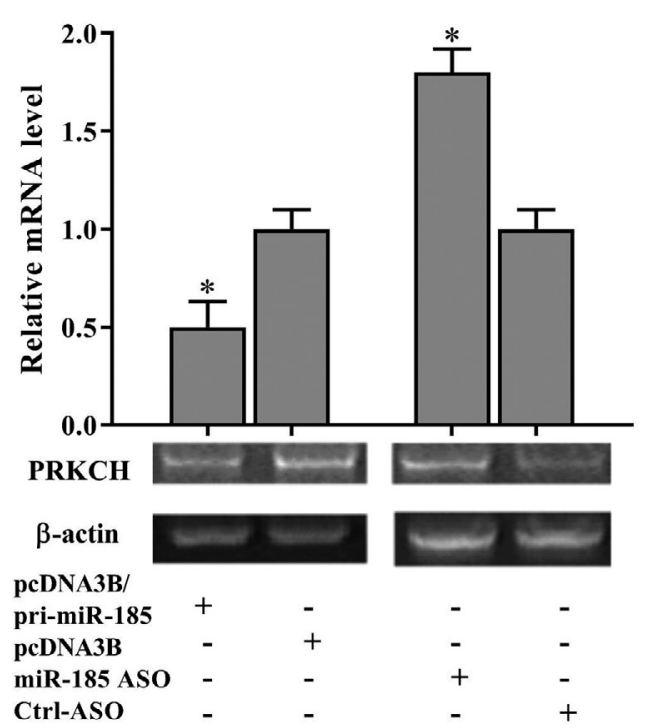

(b)

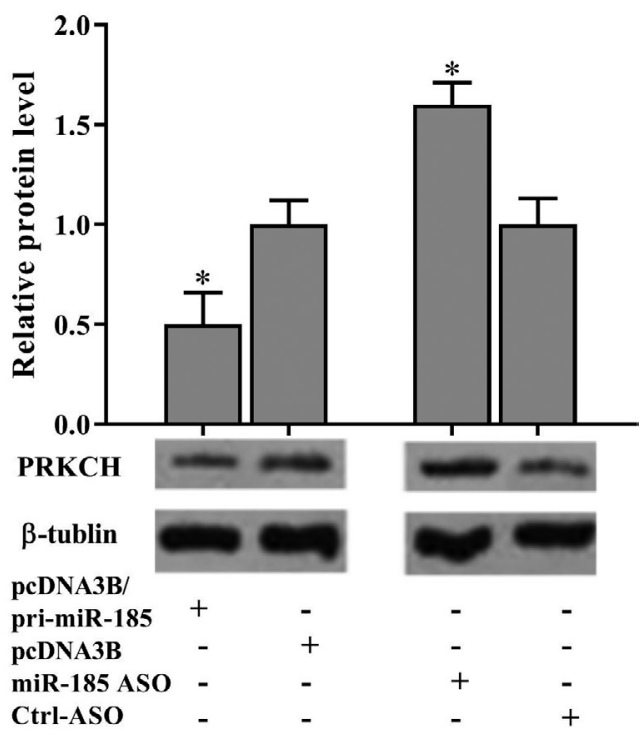

Fig. 4

Regulation of PRKCH expression by miR-185 in HepG2 2.2.15 cells

HepG2 2.2.15 cells were transfected with miR-185 ASO or control ASO or pcDNA3B/Pri-miR-185 or control vector. At $48 \mathrm{~h}$ post transfection, mRNA level of PRKCH was determined by semi-quantitative RT-PCR (a) and western blot analysis of protein level of PRKCH (b) in HepG2 2.2 .15 cells. Data were expressed as mean $\pm \mathrm{SD}(\mathrm{n}=3)$. ${ }^{*} \mathrm{P}<0.05$ versus control.

ing the miRNA complementary sequence in 3'-UTR of target mRNAs, especially the miRNA "seed region" (Jafri et al., 2017). Sequence analysis indicated that miR-185 interfered with PRKCH mRNA via direct interaction with 3'-UTR. Prediction results were confirmed by fluorescent reporter assay, which identified the direct targets for miRNAs. These results implied that anti-HBV's effect of miR-185 is partly through PRKCH. PRKCH is a member of PKC family (Newton et al., 2018), which represents a family of serin/threonine kinases, which regulate cell growth, differentiation, and transformation (Park et al., 2014). PRKCH is expressed specifically in the cell nucleus. Phosphorylation modulates the activity of many proteins that interact with nucleic acids, including DNA and RNA polymerases (Geribaldi-Doldán et al., 2019). HBx protein is important during the replicative cycle of HBV virus. Phosphorylation of HBx protein by PRKCH enhanced the activity of reverse transcriptase in vitro (Lee et al., 2012). HBx protein is a dual-specificity activator of transcription that stimulates signal transduction pathways in cytoplasm and transcription factors in nucleus when expressed in culture cell lines. In the cytoplasm, HBx stimulates the Ras-Raf-mitogen-activated protein kinase cascade, which is essential for the activation of the transcription factor AP-1. HBx activation of JNK and sustained activation of c-jun might play a role in viral transformation and pathogenesis if present in HBV infection (Wu et al., 2016). Thus, this study provided another potential mechanism of posttranscriptional regulation of $\mathrm{PRKCH}$ expression, ultimately modulating HBV replication and HBsAg expression.

In HBV chronic infections, HBV actively infects hepatocytes and maintains a low level of virus production over a long period. Factors that determine the development of HBV chronic infection have not been fully identified. Immune tolerance to the surface protein of HBV might be one of the factors involved in the development of the carrier state. This study suggested that miRNAs regulate HBV replication and maintain a low level of virus production, which may contribute to persistent HBV infection.

miRNAs play a role in antiviral defense in plants and invertebrates. Bandiera and colleagues showed that miRNAs can play a role in mammalian antiviral defense against HCV and can be induced by IFN system (Bandiera et al.,2015). Meanwhile, Pan and colleagues demonstrated that HIV used miRNA-mediated downregulation of viral protein expression to its own advantage (Pan et al., 2014). Results of the abovementioned previous studies indicated that there is an intricate physiological interplay between the cell's miRNA machinery and the invading virus.

We provided evidence that human miRNAs can inhibit HBV replication by targeting important cellular proteins. This work revealed additional information on the intricate physiological interplay between the cell's miRNAs and HBV replication. This study reported human miRNAs that can target host cell gene including PRKCH, thereby 
delaying the disease progression. In another study, miR185-5p decreased HBV S1p activity by targeting ELK1 (Fan et al., 2018). MiRNA-mimics and miRNA anti-sense constructs have potential roles in designing new generation of antiviral drugs, although many obstacles remain.

Acknowledgments. This work was supported by National Natural Science Foundation of China (81201281, H1904), the Science and Technology Research and Development Programme of Tangshan, Hebei, China (19130204C) and Scientific Research Foundation of North China University of Science and Technology (Z201607).

\section{References}

Agarwal V, Bell GW, Nam JW, Bartel DP (2015): Predicting effective microRNA target sites in mammalian mRNAs. eLife 4, e05005. https://doi.org/10.7554/eLife.05005

Arzumanyan A, Reis HMGPV, Feitelson MA (2013): Pathogenic mechanisms in HBV- and HCV-associated hepatocellular carcinoma. Nature Rev. Cancer 13,123-135. https:// doi.org/10.1038/nrc3449

Bandiera S, Pfeffer S, Baumert TF, Zeisel MB (2015): MiR-122-a key factor and therapeutic target in liver disease. J. Hepatol. 62, 448-457. https://doi.org/10.1016/j.jhep. 2014.10.004

Bertoli G, Cava C, Castiglioni I (2015): MicroRNAs: New biomarkers for diagnosis, prognosis, therapy prediction and therapeutic tools for breast cancer. Theranostics 5 , 1122-1143.http://www.thno.org/v05p1122.htm https:// doi.org/10.7150/thno.11543

Dong W, Li B, Wang J, Song Y, Zhang Z, Fu C (2017): MicroRNA-337 inhibits cell proliferation and invasion of cervical cancer through directly targeting specificity protein 1. Tumor Biol.39,101042831771132. https://doi. org/10.1177/1010428317711323

Gamazon ER, Im H-K, Duan S, Lussier YA, Cox NJ, Dolan ME, Zhang W (2010): ExprTarget: An integrative approach to predicting human microRNA targets. PLoS one 5, e13534. https://doi.org/10.1371/journal.pone.0013534

Fan HX, Feng YJ, Zhao XP, He YZ, Tang H (2018): MiR-185-5p suppresses HBV gene expression by targeting ELK1 in hepatoma carcinoma cells. Life Sciences 213, 9-17. https://doi.org/10.1016/i.lfs.2018.10.016

Fan HX, Lv P, Lv J, Zhao XP, Liu M, Zhang GL, Tang H (2016): MiR-370 suppresses HBV gene expression and replication by targeting nuclear factor IA. J. Med. Virol. 89, 834-844. https://doi.org/10.1002/jmv.24695

Geribaldi-Doldán N, Gómez-Oliva R, Domínguez-García S, Nunez-Abades P, Castro C (2019): Protein kinase C: targets to regenerate brain injuries? Front. Cell. Dev. Biol. 7,39. https://doi.org/10.3389/fcell.2019.00039

Ghoshal B, Sanfacon H (2015): Symptom recovery in virusinfected plants: Revisiting the role of RNA silencing mechanisms. Virology 479-480, 167-179. https://doi. org/10.1016/j.virol.2015.01.008
Hausser J, Zavolan M (2014) Identification and consequences of miRNA-target interactions--beyond repression of gene expression. Nature Rev. Genetics 15, 702-702. https:// doi.org/10.1038/nrg3827

Huang Y, Liu X, Liao Y, Liao Y, Zou D, Wei X, Huang Q, Wu Y (2018): Role of miR-34c in the cognitive function of epileptic rats induced by pentylenetetrazol. Mol. Med. Rep. 17, 4173-4180. https://doi.org/10.3892/mmr.2018.8441

Hong J-S, Kim N-H, Choi C-Y, Lee Y-S, Na D, Chun T, Lee YS (2015): Changes in cellular microRNA expression induced by porcine circovirus type 2-encoded proteins. Vet. Res. 46, 39. https://doi.org/10.1186/s13567-015-0172-5

Jafri MA, Al-Qahtani MH, Shay JW (2017): Role of miRNAs in human cancer metastasis: Implications for therapeutic intervention. Semin. Cancer. Biol. 44, 117-131. https:// doi.org/10.1016/j.semcancer.2017.02.004

Khairkhah N, Namvar A, Kardani K, Bolhassani A (2018): Prediction of cross-clade HIV-1 T-cell epitopes using immunoinformatics analysis. Proteins 86, 1284-1293. https://doi.org/10.1002/prot.25609

Koch A, Kogel K H(2014): New wind in the sails: improving the agronomic value of crop plants through RNAi-mediated gene silencing. Plant Biotechnol. J. 12, 821-831. https://doi.org/10.1111/pbi.12226

Lau C-C, Sun T, Ching AKK, He M, Li J-W, Wong AM, Co NN, Chan AWH, Li P-S, Lung RWM, Tong JHM, Lai PBS, Chan HLY, To K-F, Chan T-F, Wong N (2014): Viral-Human Chimeric Transcript Predisposes Risk to Liver Cancer Development and Progression. Cancer Cell 25,335-349. https:// doi.org/10.1016/i.ccr.2014.01.030

Lecellier CH, Dunoyer P, Arar K, Lehmann-Che J, Eyquem S, Himber C, Saib A, Voinnet O (2005): A cellular microRNA mediates antiviral defense in human cells. Science (New York), NY 308, 557-560. https://doi.org/10.1126/ science.1108784

Lee SH, Cha EJ, Lim JE, Kwon SH, Kim DH, Cho H, Han KH (2012): Structural characterization of an intrinsically unfolded mini-HBX protein from hepatitis B virus. Mol. Cells 34,165-169. https://doi.org/10.1007/s10059-012-0060-z

Li X, Liu X, Xu W, Zhou P, Gao P, Jiang S, Lobie PE, Zhu T (2013): C-MYC-regulated miR-23a/24-2/27a cluster promotes mammary carcinoma cell invasion and hepatic metastasis by targeting Sprouty2. J. Biol. Chem. 288, 18121-18133. https://doi.org/10.1074/jbc.M113.478560

Liu Y J, Li W, Chang F, Liu JN, Lin JX, Chen DX (2018): MicroRNA-505 is downregulated in human osteosarcoma and regulates cell proliferation, migration and invasion. Oncol. Rep. 39, 491-500. https://doi.org/10.3892/ or.2017.6142

Miyaki S, Nakasa T, Otsuki S, Grogan SP, Higashiyama R, Inoue A, Kato Y, Sato T, Lotz MK, Asahara H (2014): MicroRNA-140 is expressed in differentiated human articular chondrocytes and modulates interleukin-1 responses. Osteoarthr. Cartil. 60, 2723-2730. https:// doi.org/10.1002/art.24745

Nan A, Chen L, Zhang N, Liu Z1, Yang T, Wang Z, Yang C, Jiang Y (2017): A novel regulatory network among LncRpa, CircRar1, MiR-671and apoptotic genes promotes lead- 
induced neuronal cell apoptosis. Arch. Toxicol. 91, 1671-1684. https://doi.org/10.1007/s00204-016-1837-1

Newton AC (2018): Protein kinase C: Perfectly balanced. Crit. Rev. Biochem. Mol. Biol. 53, 208-230. https://doi.org/1 $\underline{0.1080 / 10409238.2018 .1442408}$

O'Connell D, Liang C (2016): Autophagy interaction with herpes simplex virus type-1 infection. Autophagy 12,1-9. https://doi.org/10.1080/15548627.2016.1139262

Pan D, Flores O, Umbach J, Pesola JM, Bentley P, Rosato PC, Leib DA, Cullen BR, Coen DM (2014): A neuron-specific host microRNA targets herpes simplex virus-1 ICP0 expression and promotes latency. Cell Host Microbe 15,446-456. https://doi.org/10.1016/j.chom.2014.03.004

Park GB, Choi Y, Kim YS, Lee HK, Kim D, Hur DY (2014): Silenc-

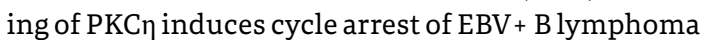
cells by upregulating expression of p38-MAPK/TAp73/ GADD45 $\alpha$ and increases susceptibility to chemotherapeutic agents. Cancer Lett. 350, 5-14. https://doi. org/10.1016/i.canlet.2014.04.020

Smirnova L, Gräfe A, Seiler A, Schumacher S, Nitsch R, Wulczyn FG (2015): Regulation of miRNA expression during neural cell specification. E. J. Neurosci. 21, 1469-1477. https://doi.org/10.1111/j.1460-9568.2005.03978.x

Takaki Y, Saito Y, Takasugi A, Toshimitsu K, Yamada S, Muramatsu T, Kimura M, Sugiyama K, Suzuki H, Arai E, Ojima H, Kanai Y, Saito H (2015): Silencing of microRNA-122 is an early event during hepatocarcinogenesis from nonalcoholic steatohepatitis. Cancer Sci. 105, 1254-1260. https://doi.org/10.1111/cas.12498

Titov II, Vorozheykin PS (2018): Comparing miRNA structure of mirtrons and non-mirtrons. Bmc Genomics 19(Suppl.3), 114. https://doi.org/10.1186/s12864-018-4473-8

Ueda T, Volinia S, Okumura H, Shimizu M, Taccioli C, Rossi S, Alder H, Liu CG, Oue N, Yasui W, Yoshida K, Sasaki H, Nomura S, Seto Y, Kaminishi M, Calin GA, Croce CM (2010): Relation between microRNA expression and progression and prognosis of gastric cancer: a microRNA expression analysis. Lancet Oncol. 11, 136-146. https://doi.org/10.1016/S1470-2045(09)70343-2
Wang M, Yin H, Zhou Y, Han J, He T, Cui L, Ai S (2018): Photoelectrochemical biosensor for microRNA detection based on multiple amplification strategies. Microchim. Acta 185, 257. https://doi.org/10.1007/s00604-018-2808-4

Wang YL, Zhou JW, Chen YL, Wang CH, Wu EY, Fu L, Xie C (2018): Quantification of distinct let-7 microRNA family members by a modified stem-loop RT-qPCR. Mol. Med. Rep. 17,3690-3696. https://doi.org/10.3892/mmr.2017.8297

Wei X, Tan C, Tang C, Ren G, Xiang T, Qiu Z, Liu R, Wu Z (2013): Epigenetic repression of miR-132 expression by the hepatitis $B$ virus $x$ protein in hepatitis $B$ virus-related hepatocellular carcinoma. Cellular Signalling 25, 1037-1043.https://doi.org/10.1016/j.cellsig.2013.01.019

Wu YH, Ai X, Liu FY, Liang HF, Zhang BX, Chen XP (2016): c-Jun $\mathrm{N}$-terminal kinase inhibitor favors transforming growth factor- $\beta$ to antagonize hepatitis B virus $X$ proteininduced cell growth promotion in hepatocellular carcinoma. Mol. Med. Rep. 13, 1345-1352. https://doi. org $/ 10.3892 / \mathrm{mmr} .2015 .4644$

Zhang GL, Li YX, Zheng SQ, Liu M, Li X, Tang H (2010): Suppression of hepatitis B virus replication by microRNA-199a$3 p$ and microRNA-210. Antiviral Res. 88, 169-175. https://doi.org/10.1016/j.antiviral.2010.08.008

Zhang MX, Zhang J, Zhang H, Tang H (2016): miR-24-3p Suppresses malignant behavior of lacrimal adenoid cystic carcinoma by targeting PRKCH to regulate p53/p21 pathway. PLoSOne 11,e0158433.https://doi.org/10.1371/ journal.pone.0158433

Zhi Q, Zhu J, Guo X, He S, Xue X, Zhou J, Hu B, Li H, Chen S, Zhao H, Kuang Y (2013): Metastasis-related miR-185 is a potential prognostic biomarker for hepatocellular carcinoma in early stage. Biomed. Pharmacother. 67, 393-398. https://doi.org/10.1016/j.biopha.2013.03.022

Zhou L, Liu SN, Han M, Ma YH, Feng SH, Zhao J, Lu HP, Yuan XX, Cheng J (2018): miR-185 inhibits fibrogenic activation of hepatic stellate cells and prevents liver fibrosis. Mol. Ther. 10, 91-102.https://doi.org/10.1016/j. omtn.2017.11.010 